\title{
Commensurability effects in overlap Josephson junctions coupled with a magnetic dots array
}

\author{
S. N. Vdovichev, S. A. Gusev, Yu. N. Nozdrin, A. V. Samokhvalov, and A. A. Fraerman \\ Institute for Physics of Microstructures, Russian Academy of Sciences, 603950, Nizhny Novgorod, GSP-105, Russia \\ E. Il'ichev, R. Stolz, and L.Fritzsch \\ Institute for Physical High Technology, P.O. Box 100239, 07702 Jena, Germany
}

(Dated: October 1, 2018)

\begin{abstract}
Experimental observation of the strong influence of an array of ferromagnetic nanodots on the critical current of a short overlap Josephson junction is reported. Pronounced commensurability effects are detected due to the presence of the additional peaks in the magnetic field induced diffraction pattern. The changes in the Fraunhofer pattern of the Josephson junctions are account for by the formation of Abrikosov vortices trapped in the electrodes which induce a phase inhomogeneity in the junction area.
\end{abstract}

PACS numbers: 74.50.+r; 75.75.+a

The role played by inhomogeneities in long Josephson junctions is known. In typical experimental realizations, periodic defects of the barrier thickness provide for a spatial modulation of the critical current density $j_{c}$ which results in sharp peaks of the critical current $I_{c}$ on variation of the external magnetic field $H[1]$. These ordered peak structures are observed at fields $H$ for which the number of enclosed magnetic flux quanta in the junction is an integer multiple of the number of defects, which is known as commensurability.

Over the last years, Josephson junctions with phase inhomogeneities have been intensively investigated. In earlier studies the properties of Josephson junctions with Abrikosov vortices (AVs) pinned in the vicinity of the barrier were discussed by several authors [2, 3]. Recently investigations of unconventional Josephson junctions with a spatially alternating sign of the critical current have attracted renewed attention. The presence of Josephson phase discontinuities results in an unusual current-phase relation [4, a highly anomalous nonFraunhofer $I_{c}(H)$ dependence [5], and a spontaneous generation of fractional Josephson vortices [6]. Although regular commensurability peaks similar to the found in 1] are quite expected for unconventional junctions, observing them is difficult due to the effect of randomness in the spatial distribution of $j_{c}$.

On the other hand, superconducting (SC) thin films coupled with arrays of magnetic nanodots show clear commensurability effects in the measurements of magnetization, critical current, and resistivity [7]. Enhanced pinning effects have already been demonstrated when the AV lattice and the magnetic dots array were matched. However, in real SC films the effects are hidden by a strong intrinsic pinning and are observable only in a narrow temperature range near $T_{C}$.

Therefore, it is quite natural to combine these activities, namely to attach an array of magnetic dots to a Josephson junction. Indeed, recently such hybrid fer-

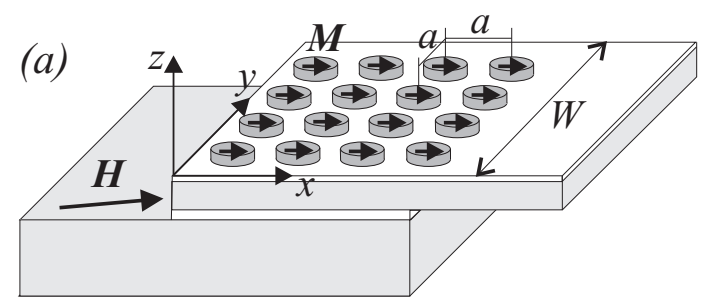

(b)

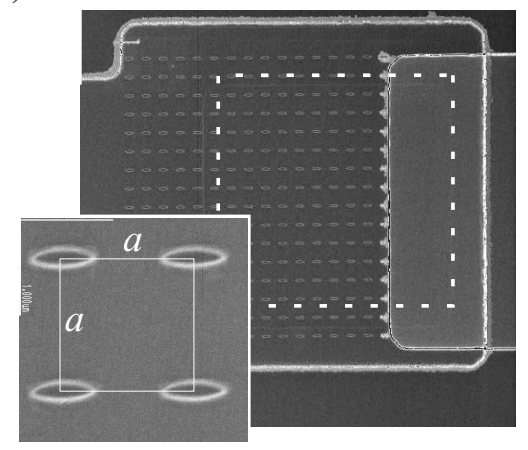

FIG. 1: (a) A regular square array of elongated magnetic particles on top of the SC electrode of the overlap Josephson junction. (b) SEM image (top view) of the setup. The dashed line indicates the junction region. The thick top electrode is shown by grey. The inset shows the elementary cell of the dots array.

romagnet/superconductor (FS) systems have been successfully prepared and studied. The system consists of a Josephson junction, formed by an edge contact of two SC films and coupled to a chain of magnetic nanodots [8]. The stray magnetic fields of the dots partially penetrate into the junction region and induce a spatially modulated Josephson phase difference $\varphi$. As a result, we have observed strong dependence of the diffraction pattern $I_{c}(H)$ on the preliminary magnetizaion of the particles.

In this Letter, we propose and implement a hybrid FS 


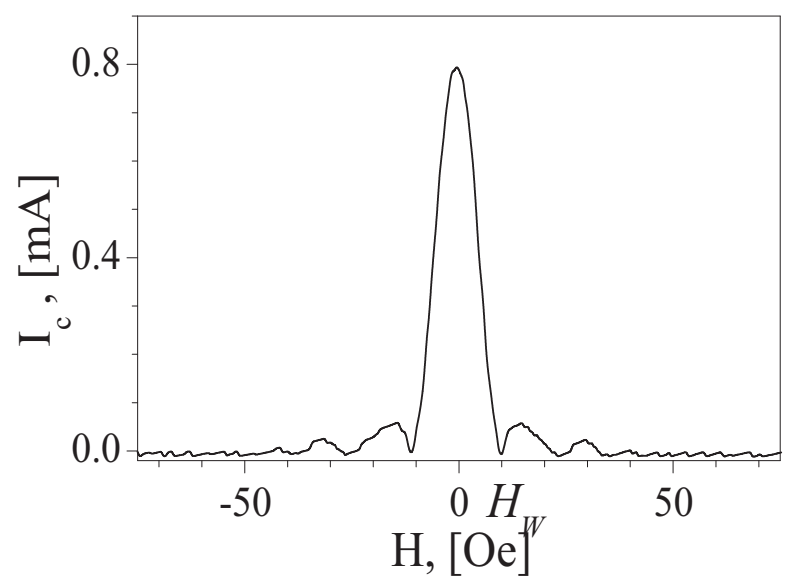

FIG. 2: Dependence $I_{c}(H)$ for the junction without the magnetic dots.

system which allows to study commensurability effects in short unconventional Josephson junctions. In this context the term "unconventional" means: these junctions exhibit a phase variation along a contact on a scale which is much smaller than the Josephson penetration depth. Due to a strictly periodic Josephson phase modulation, we succeeded in observing pronounced commensurability peaks in $I_{c}(H)$ dependence.

The investigated short overlap Josephson junction coupled with a magnetic dots array is shown in Fig.1a. The dots are separated from the top SC electrode by an insulating layer, which ensures no proximity effect and that the interaction between the junction and the dots has only magnetic character. Since the area occupied by the dots and the barrier region are far from each other and are separated by thick SC layer, we can neglect the penetration of the stray field of the dots through the edges of the junction. On the other hand, magnetic fields that reach the barrier by means of Meissner screening supercurrents does not create a Josephson phase difference. So, modulation of the Josephson phase like considered in Ref. 8] can be omitted.

The basic idea of the experiment is to form in the top SC electrode a lattice of AVs by cooling the junction through the transition temperature $T_{C}$ in the magnetic field of an array of magnetic dipoles consisting of singledomain magnetic dots with in-plane magnetization. Single flux quanta with opposite polarity are induced in the SC film at the north and south poles of the each magnetic dot and can be considered as a vortex-antivortex pair [9]. The trapped vortex-antivortex pairs generate a built-in magnetic field in the barrier, which induces a Josephson phase difference.

For our experiments, a series of $\mathrm{Nb} / \mathrm{Al}-\mathrm{AlO}_{\mathrm{x}} / \mathrm{Nb}$ overlap junctions were fabricated, employing conven- tional technology [10] with the area $W^{2} \sim 20 \times 20 \mu \mathrm{m}^{2}$. The essential feature of the junctions is the thin top electrode $(\mathrm{Nb})$ with a thickness is about $30 \mathrm{~nm}$, covered with a $50 \mathrm{~nm} \mathrm{SiO}$ dielectric. The junction has the critical current density of about $j_{c} \approx 100 \mathrm{~A} / \mathrm{cm}^{2}$ which corresponds to the Josephson length $\lambda_{J} \approx 25 \mu \mathrm{m}$ [1]. On top, the square array (lattice period $a=1.4 \mu \mathrm{m}$ ) of elongated Co nanodots was fabricated by magnetron sputtering and electron beam lithography [12]. Figure 1b shows a secondary electron microscopy (SEM) image of the system under study. The Co dots have lateral dimensions of $650 \mathrm{~nm}$ (easy axis) $\times 280 \mathrm{~nm}$ and a thickness of $55 \mathrm{~nm}$. The magnetic state of the dots was monitored by magnetic force microscopy (MFM) at room temperature. After magnetization along the easy axis all dots are in an uniformly magnetized remanent ground state. The inset of Fig. 3 shows the MFM image of the array of preliminarily magnetized nanodots. The dipole stray fields characteristics are clearly visible. A minor variation in the contrast for different particles is explained by the small tilt angle between sample and scanning planes. Demagnetization converts these magnetic particles into a multivortex state. Measurements of the critical current $I_{c}$ as a function of the external magnetic field $H$, applied in the plane of the junction, were made using computercontrolled current sweeps at $4.2 \mathrm{~K}$. The criterion for $I_{c}$ is the detection of a voltage, typically about $5 \mu \mathrm{V}$.

Figure 2 shows the ordinary Fraunhofer dependence $I_{c}(H)$ of the Josephson junction before the magnetic particles were sputtered: there is the critical current maximum at zero field and oscillations of $I_{c}$ which diminish in amplitude with increasing field $H$. The measured period of the diffraction pattern $H_{W}=\Phi_{0} / \Lambda W \simeq 11.3$ Oe enables the determination of the effective depth of the magnetic field penetration into the $\mathrm{SC}$ electrodes $\Lambda \approx 0.1 \mu \mathrm{m}$, where $\Phi_{0}$ is the magnetic flux quantum. The same Fraunhofer dependence was observed when the magnetic dots are mainly in the vortex state.

Qualitatively a different diffraction pattern is observed if the array of the uniformly magnetized nanodots is attached to the junction. Figure 3 shows the fielddependent critical current $I_{c}(H)$ of the junction coupled with the ordered lattice of magnetic dipoles. One can see that the typical value of the Josephson current through junction is noticeably suppressed in comparison with the case before. The symmetry of the diffraction pattern with respect to the external magnetic field inversion is broken: $I_{c}(-H) \neq I_{c}(H)$. There is also a set of observable critical current maxima for high magnetic fields $|H|>H_{W}$. The two dominant side peaks of the $I_{c}(H)$ curve are clearly observed at values of the field $H_{+}=100 \mathrm{Oe}$ and $H_{-}=-60 \mathrm{Oe}$. The relative position of $H_{ \pm}$peaks correspond to the addition/removal of one flux quantum $\Phi_{0}$ per period $a$, and the following commensurability condi- 


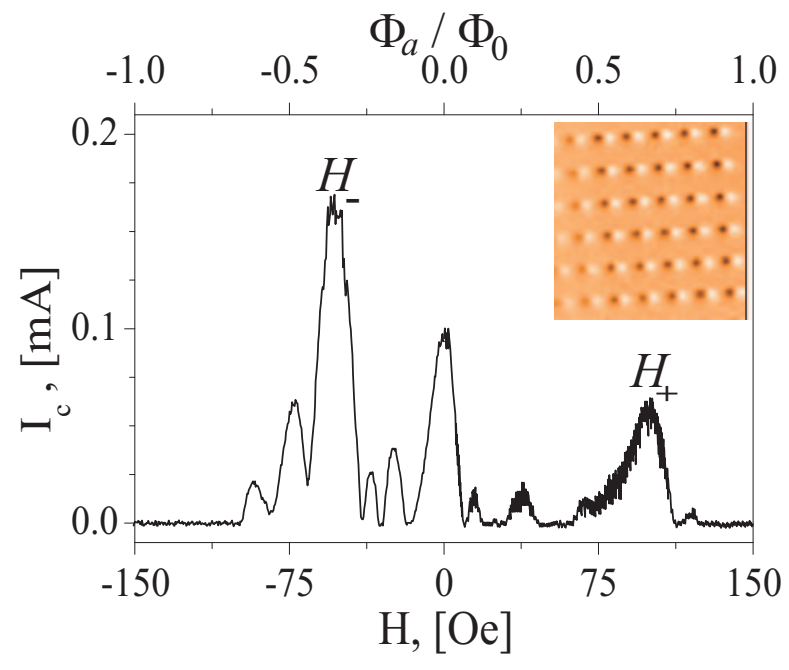

FIG. 3: Dependence $I_{c}(H)$ for junction coupled with the array of the uniformly magnetized nanodots. The insert shows the MFM images of the part $10 \times 10 \mu \mathrm{m}^{2}$ of the ordered lattice of dipoles.

tion is satisfied to a good accuracy:

$$
\Phi_{a}\left(H_{+}\right)-\Phi_{a}\left(H_{-}\right) \approx \Phi_{0},
$$

where $\Phi_{a}(H)=H S_{a}$ is the value of the magnetic flux of the field $H$ through an elementary cell $S_{a}=\Lambda \times a$.

The studied system can be represented by a square Josephson junction in which the gauge-invariant phase difference $\varphi(\mathbf{r})$ depends on a two-dimensional vector $\mathbf{r}=$ $(x, y)$ lying in the junction plane. The junction occupies the area $0 \leq x, y \leq W$. The size $W$ is small in comparison with the Josephson penetration depth $\left(W<\lambda_{J}\right)$, and self-field effects of the Josephson current are negligible. Magnetostatic calculations show that for a typical value of the saturation magnetization $M_{s}=800$ Oe and for the parameters taken from the experiments, the stray field of both poles of the in-plane magnetized dot creates a (positive or negative) flux $\Phi_{s}>\Phi_{0}$ through the surface of the top SC electrode. So, in accordance with the criterion proposed in [9], we assume that each magnetic dot creates a pair of opposite vortices which pierce the top electrode of the junction. The phase difference $\varphi^{M}(\mathbf{r})$, created by the array of magnetic dots is determined only by the positions of vortices trapped in the top electrode of the junction, and obeys the following equations:

$$
\begin{aligned}
\triangle \varphi^{M} & =0 \\
\operatorname{curl}_{z}\left(\nabla \varphi^{M}\right) & =2 \pi \sum_{n, m}\left[\delta\left(\mathbf{r}-\mathbf{r}_{n m}^{+}\right)-\delta\left(\mathbf{r}-\mathbf{r}_{n m}^{-}\right)\right]
\end{aligned}
$$

with the following boundary conditions at the edges of the junction:

$$
\left.\partial_{x} \varphi^{M}\right|_{x=0, W}=0,\left.\quad \partial_{y} \varphi^{M}\right|_{y=0, W}=0 .
$$

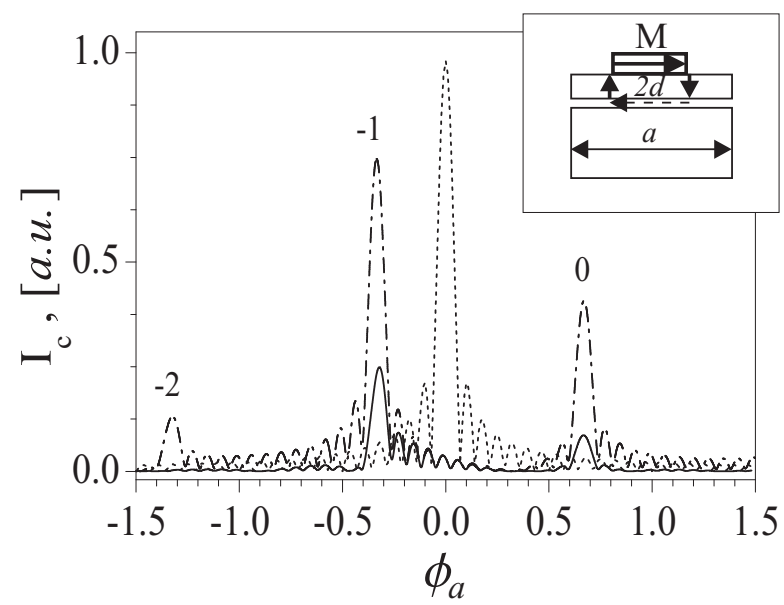

FIG. 4: Dependence of $I_{c}(H)$ for a junction when the vortexantivortex pairs form the ordered square lattice $(W=14 a$, $d=a / 3$ ). The dash-dotted curve corresponds to $\alpha=0^{\circ}$, the solid curve to $\alpha=9^{\circ}$. The dotted line represents the Fraunhofer pattern in the absence of vortex-antivortex pairs in the top electrode. The numbers near the peaks denote the corresponding values of $n$ in (7). The inset schematically shows the elementary cell of the junction with the attached magnetic dot above. The vortex-antivortex pair is represented by the vertical arrows near the opposite poles of the nanoscale magnet.

Here $\mathbf{r}_{n m}^{ \pm}=(a n \mp d, a m)$ are the coordinates of vortices $(+)$ and antivortices $(-)$ created by the square $(a \times a)$ array of the uniformly magnetized particles $\mathbf{M}=M \mathbf{x}_{0}$, having a size equal to $2 d$ in the easy direction [9].

The critical current across the junction is given by [13]

$$
I_{c}=j_{c} \mid \int_{0}^{W} d x d y \sin [\varphi(x, y)],
$$

where the phase difference

$$
\varphi(x, y)=\varphi^{M}(x, y)+\frac{2 \pi \Lambda}{\Phi_{0}}\left(H_{x} y-H_{y} x\right)
$$

depends both on the phase difference $\varphi^{M}$ and on the external magnetic field $\mathbf{H}=H_{x} \mathbf{x}_{0}+H_{y} \mathbf{y}_{0}$ applied in the junction plane. By using the described model, we performed simulations of the dependence of the critical current $I_{c}$ on the external magnetic field $\mathbf{H}$ for various values of the relation $d / a$ and the different values of the angle $\alpha$ between the $\mathbf{H}$ and $\mathbf{M}$ direction. Figure 4 shows the results of a simulation of the diffraction pattern $I_{c}\left(\phi_{a}\right)$ $\left(\phi_{a}=\Phi_{a}\left(H_{x}\right) / \Phi_{0}\right)$ for the parameters $W=14 a$ and $d=a / 3$, which are close to those of the samples in use. It is seen that $I_{c}\left(\phi_{a}\right)$ oscillates with a slightly varying amplitude when the flux $\phi_{a}$ increases. The period of the oscillations $\triangle \phi_{a}$ is determined by the junction width $W$ : $\triangle \phi_{a}=a / W$. Strong additional peaks occur at

$$
\phi_{a}[n]=n+\phi_{d}, \quad n=0, \pm 1, \pm 2 \ldots,
$$


where $\phi_{d}=2 d / a$. One observes that the positions of the two dominant peaks calculated from the condition (7) for $n=-1,0$ and $d=a / 3\left(\phi_{a}[-1] \simeq-0.33, \phi_{a}[0] \simeq 0.66\right)$ is in good agreement with the experimental data (see Fig. 3 to compare). The fixed shift of the peaks $\phi_{d}$ can be explained by the presence of the built-in field $\mathbf{H}_{i n}=$ $H_{\text {in }} \mathbf{x}_{0}=-H_{a}(2 d / a) \mathbf{x}_{0}$, which creates the average gradient of the phase difference: $\left\langle\partial_{y} \varphi^{M}\right\rangle=-q_{a}(2 d / a)$. Here $H_{a}=\Phi_{0} / S_{a}$ is the field of one flux quantum enclosed in the cell area, and $q_{a}=2 \pi / a$ is the inverse lattice constant. The peak for $n=0$ occurs when the $x$-projection of the external magnetic field $\mathbf{H}$ overcomes the built-in field $\mathbf{H}_{\text {in }}: H_{x}+H_{\text {in }}=0$.

The position of the dominant peaks is determined by commensurability between the periodic modulation of the Josephson current due to magnetic dots and the imposed modulation owing to the external magnetic field H. The maximum contribution from the oscillating term in $j=j_{c} \sin [\varphi(x, y)]$ to the total current across the junction $I$ corresponds to the field $\mathbf{H}$ for which the the spatial Josephson current wave

$$
j \sim \sin (\mathbf{q} \mathbf{r}), \quad \mathbf{q}=\left(q_{x}, q_{y}\right)=\frac{2 \pi \Lambda}{\Phi_{0}}\left(\mathbf{H}+\mathbf{H}_{i n}\right)
$$

has the same space harmonics as the phase factor $\varphi^{M}(x, y)$. If the angle $\alpha$ is small so as $q_{y} \ll q_{a}$, the resonant condition between the wave number $q_{x}$ and the inverse lattice constant $q_{a}$

$$
q_{x}=n q_{a}, \quad n=0, \pm 1, \pm 2 \ldots
$$

defines the values of the external magnetic field $H_{x}$ for which the commensurability peaks of $I_{c}(H)$ arise:

$$
H_{x}=n H_{a}-H_{i n}
$$

By taking into account that $\phi_{a}=H_{x} / H_{a}$, it is easy to rewrite the last equation in the form of condition (7). Note that the considerable suppression of the characteristic critical current observed in the experiments can be explained by misalignment of the external magnetic field $\mathbf{H}$ with respect to the easy axis of the magnetic dots.

So, most of the qualitative peculiarities of the experimentally observed diffraction pattern can be consistently explained in the framework of the model, when the array of the uniformly magnetized nanodots creates an ordered lattice of vortex-antivortex pairs in the top electrode of the junction. However, the critical current dependence $I_{c}(H)$ in the vicinity of $H=0$ (see Fig. 3) is not consistent with the result of the simulations presented at Fig. 4. This disagreement between theory and experiment can be explained by the junction design. There is a certain area near the edge of the junction with a thick top electrode (about $200 \mathrm{~nm}$ ), when the magnetic dots and, thus, a periodic modulation of the phase difference are absent. This part of the junction has an ordinary Fraunhofer pattern like shown in Fig. 2, and is responsible for the central peak of $I_{c}(H)$ in Fig. 3.
In summary, we measured commensurability peaks in $I_{c}(H)$ of the short overlap Josephson junctions coupled to an array of the magnetic nanodots. The position and the amplitude of the observed peaks conform to the proposed model in which the vortex-antivortex pair are created by cooling the junction through the transition temperature $T_{c}$ in the stray magnetic fields of the in-plane magnetized dots. Thus, we have made the indirect confirmation that single flux quanta with opposite polarity are induced in the SC layer at the opposite poles of the in-plane magnetic dot [9]. The experimental results show that the vortex lattice is quite perfect despite of the intrinsic pinning inherent in SC films. We observed a strong variation of the maximum critical current which depends on the magnetic state of the particles. This gives us the possibility to control the Josephson phase in the hybrid FS systems.

We are indebted to A.S. Mel'nikov and I.A. Shereshevskii for useful discussions, and to G.L. Pakhomov and B.A. Gribkov for assistance in the preparation of the samples and MFM measurements. The work was supported by the RFBR (\#03-02-16774) and the program RAS "Quantum Macrophysics".

[1] A.A. Golubov, I.L. Serpuchenko, and A.V. Ustinov, Zh. Eksp. Teor. Fiz. 94, 297 (1988) [Sov.Phys. - JETP 67, 1256 (1988)]; M.A. Itzler and M. Tinkham, Phys. Rev. B 51, 435 (1995).

[2] S.L. Miller,K.R. Biagi, J.R. Clem, and D.K. Finnemore, Phys. Rev. B, 31, 2684 (1985); A.V. Ustinov et al., Phys. Rev. B, 47, 944 (1993); M.V. Fistul, G.F. Giuliani, Phys. Rev. B 58, 9348 (1998).

[3] A.A. Golubov, M.Yu. Kupriyanov, Zh. Eksp. Teor. Fiz. 92, 1512 (1987)[Sov.Phys. - JETP 65, 849 (1987)].

[4] A.A. Golubov, M.Yu. Kupriyanov, E. Il'ichev, Rev. Mod. Phys. 76, 411 (2004); A. Buzdin, A.E. Koshelev, Phys. Rev. B 67, 220504(R) (2003).

[5] H. Hilgenkamp, J. Mannhart, and B. Mayer, Phys. Rev. B 53, 14586 (1996); H.J.H. Smilde et al., Phys. Rev. Lett. 88, 057004 (2002); R.G. Mints, V.G. Kogan, Phys. Rev. B 55, R8682 (1997);

[6] R.G. Mints et al., Phys. Rev. Lett. 89, 067004 (2002); H. Hilgenkamp et al., Nature (London) 422, 50 (2003). E. Goldobin, A. Sterck, T. Gaber, D. Koelle, R. Kleiner, Phys. Rev. Lett. 92, 057005 (2004).

[7] J.I. Martin, M. Velez, J. Nogues, and I.K. Schuller, Phys. Rev. Lett. 79, 1929 (1997); D.J. Morgan, and J.B. Ketterson, Phys. Rev. Lett. 80, 3614 (1998); M.J. Van Bael, K. Temst, V.V. Moshchalkov, Y. Bruynseraede, Phys. Rev. B 59, 14674 (1999).

[8] A.Y. Aladyshkin et al., J. Magn. Magn. Mater. 258259, 406 (2003); A.V. Samokhvalov, JETP Lett. 78, 369 (2003); S.N. Vdovichev et al., JETP Lett. 80, 651 (2004).

[9] M.J. Van Bael et al., Phys. Rev. Lett. 86, 155 (2001); M.V. Milosevic, and F.M. Peeters, Phys. Rev. B 69, 104522 (2004).

[10] R. Stolz, L. Fritzsch, and H.-G. Meyer, Supercond. Sci. Technol. 12, 806 (1999). 
[11] K.K. Likharev, Dynamics of Josephson Junctions and Circuits (Gordon and Breach, New York, 1986).

[12] A.A. Fraerman et al., Phys. Rev. B 65, 64424 (2002).
[13] B.D. Josephson, Adv. Phys. 14, 419 (1965). 